\title{
Profesor Dr. Marshall Raymond Urist (1914-2001): descubridor de la formación ósea por inducción
}

\author{
Raúl Álvarez-San Martín \\ Centro Médico ABC, Ciudad de México, México
}

\begin{abstract}
Resumen
Marshall R. Urist fue un ortopedista estadounidense que trabajó en la Universidad de California en Los Ángeles. Sus trabajos de investigación sobre la formación ósea trajeron a la ciencia médica, en 1965, el descubrimiento de una sustancia inductora del crecimiento óseo conocida como proteína ósea morfogenética. Esta proteína hoy en día es una sustancia producida por ingeniería genética recombinante, que ayuda a la regeneración del tejido conectivo y estimula a las células no especializadas a transformarse hacia la línea celular osteoblástica. Este documento biográfico sobre Urist revisa su vida, carrera, artículos clásicos y contribuciones esenciales a la ciencia médica.
\end{abstract}

PALABRAS CLAVE: Proteína. Hueso. Morfogenética. Formación. Inducción.

\begin{abstract}
Marshall R. Urist was an American orthopedic surgeon who worked in University of California at Los Angeles. His scientific work about bone formation brought to medical science the discovery of a bone growth inductor substance known as bone morphogenetic protein in 1965 . The protein today is a recombinant genetically produced substance which helps to regenerate connective tissues and stimulates non specialized cells to transform in to osteoblastic cell lineage. This biographical document about Urist reviews his life, career, classic scientific papers and those landmark contributions to medical science.
\end{abstract}

KEY WORDS: Protein. Bone. Morphogenetic. Formation. Induction.

En 2004, la American Academy of Orthopaedic Surgeons (AAOS) mencionó que la vida de Marshall Urist fue el ejemplo de que la investigación clínica puede estimular avances significativos en el tratamiento médico y de que, además, puede dar origen a toda una industria relacionada. Sus contribuciones a la ortopedia son un apoyo testamentario al valor y la aplicación de la investigación clínica a la práctica médica contemporánea; sin embargo, no se queda ahí. Urist aportó una transformación científica de la cirugía ortopédica como la vemos hoy día, al participar en la alteración de la dirección curricular después de la Segunda Guerra Mundial. En 1929 y 1930, en los EE.UU. comenzaron las residencias de patología ortopédica de tiempo completo en la Mayo Clinic de Boston, el New York Orthopedic Hospital y el Hospital for the Ruptured and Crippled (en la actualidad Hospital for Special Surgery) de Nueva York, siendo la Patología la única ciencia básica en Ortopedia en los EE.UU. A partir de 1950 comenzaron a incluirse otras disciplinas científicas en los laboratorios y los departamentos de los hospitales ortopédicos, pero no fue

\section{Correspondencia:}

Raúl Álvarez-San Martín

Campus Observatorio

Torre Donald Mackenzie, Consultorio 111, Sur 136

No. 116

Col. Las Américas, Del. Álvaro Obregón

C.P. 01120, Ciudad de México, México

Fecha de recepción: 03-11-2016

Fecha de aceptación: 03-07-2017

Gac Med Mex. 2017;153:946-951

E-mail: alvarez_sanmartin@yahoo.com.mx

DOI://dx.doi.org/10.24875/GMM.17002973

Contents available at PubMed

www.gacetamedicademexico.com 
hasta 1954 que se fundó la Orthopaedic Research Society (ORS) con el fin de crear el interés exclusivo sobre la investigación científica. A partir de esta sociedad fue que Marshall Urist, desde Los Ángeles, se convirtió en uno de los jóvenes cirujanos ortopedistas (junto con Andrew Baset en Nueva York, Jonathan Cohen y Henry Mankin en Boston, y Robert D. Ray en Chicago) que revolucionaron la disciplina, pues ocuparon cargos de tiempo completo en investigación básica y administraron servicios médicos desde el laboratorio, conjuntando así a la investigación avanzada con la clínica quirúrgica hospitalaria en Ortopedia'.

El Dr. Jeffrey J. Eckardt, siendo profesor y jefe del Departamento de Cirugía Ortopédica de la Escuela de Medicina de la Universidad de California en Los Ángeles (UCLA), mencionó en el año 2013 que Marshall R. Urist fue un hombre de visión, de interminable energía, de curiosidad desenfrenada y abrumadora compasión humana ${ }^{2}$. Sin duda, Urist fue un visionario; él mismo dijo en 1997 en la revista Journal of $\mathrm{NIH}$ Research que la proteína ósea morfogenética (BMP, Bone Morphogenetic Protein) estaba destinada a llevar a la osteogénesis bajo el control de los cirujanos antes de que terminara el siglo $X X^{3}$. También en ese mismo año mencionó en un editorial del Journal of Bone and Mineral Research que era el inicio de la molecularización del conocimiento del desarrollo del sistema esquelético ${ }^{4}$.

Marshall Raymond Urist nació el 11 de junio de 1914 en Chicago, Illinois, EE.UU., pero creció en la comunidad rural de South Haven, en Michigan, al norte de Ios EE.UU. Al terminar sus estudios de bachillerato, Urist ingresó a la Universidad de Michigan, donde se graduó en 1936, para luego, en 1937, poder obtener el grado de Maestro en Ciencias en la Universidad de Chicago, después de trabajar en el laboratorio del Dr. Franklin Chambers McLean (fundador de las residencias médicas en los EE.UU.). Años más tarde, en 1941, obtuvo su grado médico al egresar de la Escuela de Medicina de la Universidad John Hopkins, en Baltimore. Después de completar un año de internado de cirugía en el Children's Hospital en Baltimore, se enroló en la Armada de los EE.UU., donde fue incluido en las actividades bélicas de la Segunda Guerra Mundial en 1943. En dicho periodo fue reclutado a la $22^{a}$ División del Hospital General en Palm Desert, California, cuya unidad fue eventualmente asignada a Inglaterra. En Europa fungió como Jefe de Ortopedia, luego como Médico Adscrito Regional del $802^{\circ}$ Grupo Hospitalario y en el servicio de Ortopedia de la $92^{a}$
División de Hospital General en Frankfurt am Main, en Alemania 5 .

Posteriormente, en 1945, fue asignado al Pentágono, donde una de sus encomiendas ocurrió en 1946 en la Oficina de Cirujanos Generales, para registrar los avances realizados durante la Segunda Guerra Mundial en materia del manejo de fracturas compuestas de la extremidad inferior, secundarias a heridas de combate y accidentes en vehículos militares $^{6-13}$. Sus trabajos resultaron en contribuciones a la publicación de un tratado sobre el manejo de las fracturas compuestas a cargo del Dr. Mather Cleveland, titulado Orthopaedic Surgery in World War II in the European Theather of Operations ${ }^{8}$, refiriéndose al término "sala de operaciones europeas" como European Theather of Operations (ETO), una zona designada para las operaciones militares americanas en la región europea. Por sus esfuerzos en este periodo recibió en 1947 varios honores, como el reconocimiento Sir Henry Wellcome Award por sus importantes aportaciones a la cirugía militar ${ }^{9}$, una Estrella de Bronce (al mérito) y dos citas, una del General Leonard Heaton y otra del General Dwight D. Eisenhower ${ }^{5}$.

Después de su carrera militar, durante ese mismo año, en el verano de 1946, Urist se incorporó como residente en el Massachusetts General Hospital, donde trabajó con el Dr. Smith-Petersen concentrado en trabajos sobre artroplastia primaria de cadera ${ }^{13}$. Luego completó su entrenamiento de residencia médica en Ortopedia en el Boston Children's Hospital, dedicándose ahí principalmente al estudio de la poliomielitis. A finales de 1947, Urist regresó a Chicago por un año para investigar la formación endóstica del hueso bajo la influencia de los estrógenos, desempeñándose además en la Universidad de Chicago como Instructor e Investigador Asociado al Departamento de Fisiología, junto a su mentor y amigo el Profesor Franklin C. McLean ${ }^{14-17}$ (Fig. 1).

En 1948 se mudó a California para establecer su práctica académica, donde comenzaría sus investigaciones sobre los efectos de los estrógenos en el hueso ${ }^{18,19}$, para luego incorporarse como profesor clínico asistente en 1952, con el apoyo del Dr. Stafford Warren, decano de la recién formada Escuela de Medicina de la UCLA. Ahí mismo fue nombrado profesor adjunto de cátedra en 1954 y luego profesor de Cirugía Ortopédica en $1969^{14,15}$ (Figs. 2 y 3).

Urist mantuvo relación estrecha con Franklin McLean, de la Universidad de Chicago, hasta que ocurrió la muerte de su mentor en 1968. En 1950, 
Urist y McLean recibieron el importante reconocimiento de la AAOS en investigación básica (Kappa Delta Award), por su trabajo sobre los efectos de los estrógenos en la formación ósea ${ }^{20,21}$.

Junto a Franklin McLean, Urist publicó en la Prensa de la Universidad de Chicago, dentro la serie editorial The Scientist Library: Biology and Medicine, tres exitosas ediciones (en 1955, 1961 y 1968) del libro titulado Bone: An Introduction to the Physiology of Skeletal Tissue ${ }^{22,23}$. En la UCLA, bajo contrato de la Comisión de Energía Atómica de los EE.UU. en el US Veterans Hospital, Urist inició sus investigaciones sobre el tratamiento del osteosarcoma con tetraciclinas marcadas con estroncio 90. También en la UCLA fundó un laboratorio específico para la investigación ósea (Bone Research Laboratory at UCLA), mejor conocido como «The Bone Lab», lugar que pronto se convertiría en centro de intercambio científico e intelectual ${ }^{24}$.

Sin embargo, su línea de investigación principal fue la fisiología del crecimiento óseo, donde uno de sus trabajos, publicado en 1965 en la revista Science y titulado Bone: Formation by Autoinduction, planteó la existencia de osteoinductores ${ }^{25}$. Según la ORS, dicho trabajo es considerado hoy en día el inicio de las investigaciones sobre el crecimiento óseo ${ }^{26}$. De hecho, el National Institute of Health Research de los EE.UU. lo catalogó en 1997 como un punto de contribución crucial para la ciencia ${ }^{26}$.

En el año 2008, el Dr. Richard A. Brand, entonces editor en jefe de la revista Clinical Orthopedics and Related Research, comentó que desde 1953 Marshal R. Urist hizo énfasis sobre el fenómeno de inducción, además de argumentar que la importancia del fenómeno fisiológico radicaba en el efecto físico-químico que un tejido ejerce sobre otro al entrar ambos en contacto ${ }^{27,28}$. También Brand mencionó que Urist postuló que la inducción parecía ser un mecanismo mucho más complejo que el efecto humoral directo de una sustancia química difusible, y que además el aislamiento o la caracterización de las sustancias inductoras a partir de los extractos no había sido aún conseguida de manera satisfactoria, y que probablemente no sería posible hacerlo sino hasta que se obtuviese a partir de experimentos científicos un conocimiento sobre el proceso de la osificación endocondral y la formación ósea endóstica ${ }^{28}$.

Urist tenía razón, y una serie de trabajos de investigación realizados por él y su equipo llevaron al descubrimiento y el aislamiento de factores de crecimiento que se denominaron BMP29-33. Un trabajo en

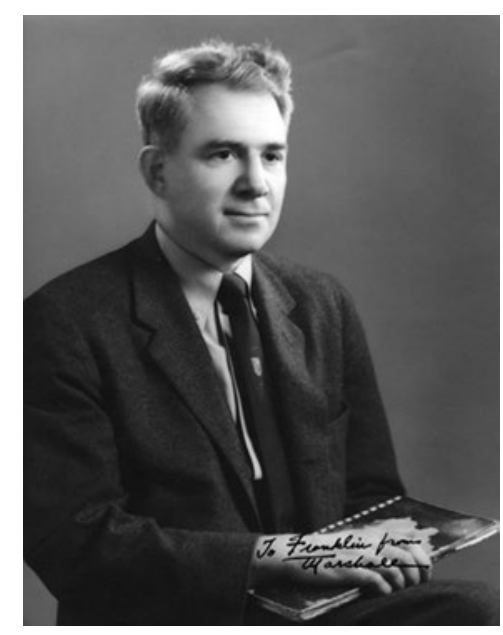

Figura 1. Marshall R. Urist, profesor de Fisiología en la Universidad de Chicago. En la leyenda: Para Franklin de Marshall. Reproducida con autorización de University of Chicago Photographic Archive, (apf108454), Special Collections Research Center, University of Chicago Library.

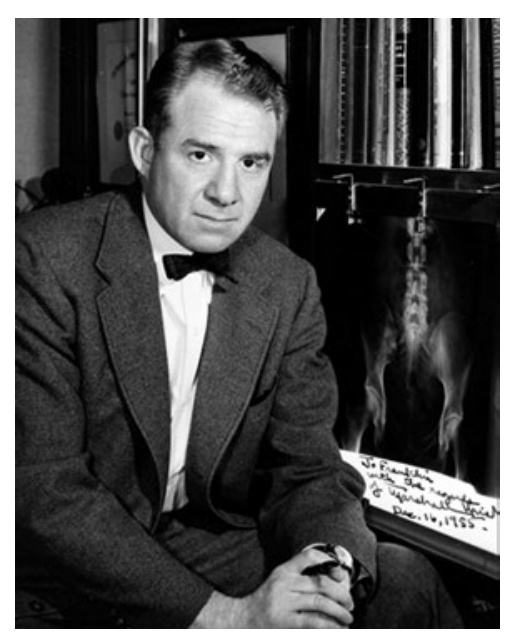

Figura 2. Marshall R. Urist, miembro de la Facultad de Medicina de la Universidad de California en Los Ángeles (UCLA). En la Leyenda: Para Franklin con los saludos de Marshall Urist. Diciembre 16, 1955. Reproducida con autorización de University of Chicago Photographic Archive, (apf1-08455), Special Collections Research Center, University of Chicago Library.

particular, que es considerado hoy un «clásico científico» por la revista Clinical Orthopedics and Related Research, titulado "A morphogenetic matrix for differentiation of cartilage in tissue culture", permitió a Urist descubrir las propiedades morfogenéticas de la matriz ósea desmineralizada, donde reportó que actúan como factores de crecimiento para la estimulación y la formación selectiva de hueso en diversos tejidos $^{31,32}$. Hasta ese momento solo se sabía que los tejidos esqueléticos cultivados in vitro como órgano embrionario estaban predestinados o prediferenciados a desarrollarse hacia modelos de tejidos 


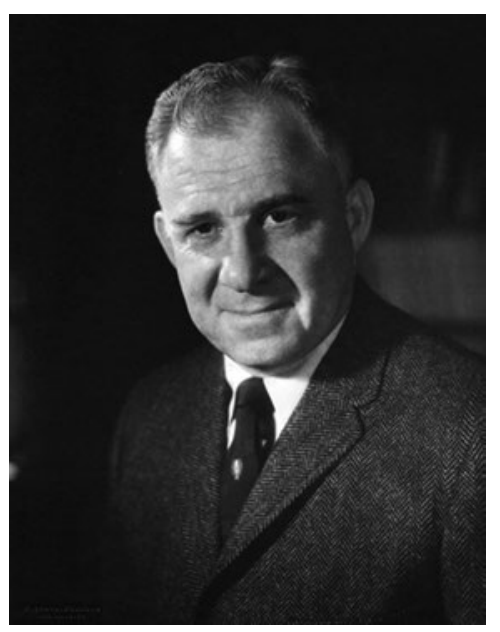

Figura 3. Marshall R. Urist, miembro de la Facultad de Medicina de la Universidad de California en Los Ángeles (UCLA). Reproducida con autorización de University of Chicago Photographic Archive, (apf108456), Special Collections Research Center, University of Chicago Library.

específicos (p. ej., cartílago o tejido fibroso), ante la falta de inducción de la diferenciación de las células mesenquimatosas posnatales, a pesar de los cambios realizados en la composición de los medios de crecimiento celular, es decir, los cambios químicos solo inducían modificaciones en el metabolismo celular, mas no en su línea de diferenciación tisular específica.

Otro estudio publicado por Urist en 1970, en un suplemento de la revista Calcified Tissue Research, titulado "A morphogenetic matrix for differentiation of bone tissue", reportó los requerimientos locales extrínsecos para que las células mesenquimatosas posfetales realicen la morfogénesis ósea ${ }^{29,30}$. En otro trabajo más, fechado en 1971 y publicado en Journal of Dentistry Research, titulado «Bone Morphogenetic Protein", Urist mencionó los progresos de investigación sobre las características bioquímicas de las proteínas morfogenéticas, incluyendo un apéndice con conceptos teóricos y de terminología relacionada ${ }^{33}$. Ambos trabajos fueron reimpresos como clásicos por la revista Clinical Orthopedics and Related Research en el año 200930,34. Urist, en «Bone Morphogenetic Protein", reportó las características ultraestructurales de una matriz ósea desmineralizada procesada, donde hizo notar que el $3 \%$ del tejido desecado y libre de grasa ocupaba un tercio del dominio de la matriz; también que estos constituyentes incluían a moléculas muy largas de proteoglicanos con uniones a fibras de colágena que se tiñeron con nitrato de bismuto, además de hacer notar una hipótesis interesante sobre el desarrollo del hueso y la médula ósea en implantes de residuos de matriz (derivados de la rotura enzimática, la extracción química o procedimientos de bloqueo orgánico), en la cual sugirió que la actividad ósea morfogenética residía en un complejo proteínico macromolecular interfibrilar ${ }^{33,35}$.

Urist continuó con sus investigaciones sobre la inducción ósea, pero no fue hasta 1983 que publicó nuevamente en la revista Science su trabajo titulado "Bone Cell differentiation and Growth Factors: Induced Activity of Chondroosteogenic DNA»36. Dicho trabajo permitió encontrar las sustancias proteínicas con capacidad osteoinductiva e inductora de diferenciación esquelética, que conforman a la familia de las BMP. En su trabajo, Urist concluyó que tanto la generación como la regeneración óseas son atribuidas a la eficiencia de las BMP y a los factores de crecimiento derivados del hueso ${ }^{36}$. Por dicha hipótesis y sus hallazgos, Marshall Urist fue incluido en la lista de nominados a recibir el Premio Nobel de Medicina y Fisiología de $1991^{24}$.

Con estos trabajos, el camino hacia el uso de las BMP se había trazado, pues aportaron a la ciencia médica el concepto de «inducción ósea»37,38. Dicho concepto surgió a partir de la implantación en músculos de animales de cantidades conocidas de matriz ósea alogénica, liofilizada y desmineralizada, para luego, a partir de dicho modelo animal, dar paso a las investigaciones sobre la fisiología y la bioquímica de la inducción ósea ${ }^{38-40}$ hasta quedar demostrada en $1970^{41}$. El término "proteína ósea morfogenética» (BMP) fue introducido en 1971 para referirse a una molécula natural de origen proteico como inductora de morfogénesis ósea ${ }^{33}$. En las décadas de 1980 y 1990 se lograrían purificar ${ }^{42-46}$ y aislar ${ }^{45,47}$ las primeras proteínas morfogenéticas humanas.

El trabajo científico de Urist fue prolífico, llegándose a registrar en el motor de búsqueda bibliográfica PubMed 486 artículos al respecto, además de realizar alrededor de 200 conferencias por todo el mundo. Por su trabajo y descubrimientos, Urist recibió diversas distinciones y premios científicos, como un segundo Delta Kappa Award de la AAOS por su trabajo publicado en la revista Science en 1983, la medalla Claude Bernard, un Fellowship de la Fundación John Simon Guggenheim y el Bristol Myers/Squibb Zimmer Distinguished Achievement Award por sus logros en la investigación ortopédica. Recibió un grado honorario en medicina por la Universidad de Lund en Suecia, un Fellowship honorario por el Royal College of Surgeons de Edimburgo, fue miembro activo y presidente de la Association of Bone and Joint Surgeons (ABJS), 
obtuvo una membresía honoraria de la Asociación Ortopédica Japonesa, y fue presidente de la Society of International Research in Orthopedic Surgery and Traumatology y de la Hip Society de los EE.UU. Fungió también como segundo editor en jefe de la prestigiada revista ortopédica Clinical Orthopedics and Related Research desde 1966 hasta 1993. A partir de 1996 se instituyó por parte de la ORS y la ABJS el «Premio Marshall R. Urist», que se encuentra patrocinado por la revista Journal of Orthopaedic Surgery con una bolsa de US $\$ 5,000$ y una placa conmemorativa, aportados por el grupo editorial Wiley BlackweII, cuya entrega se realiza en el congreso de la ORS ${ }^{48}$. Dicho premio está destinado al reconocimiento de todos aquellos que se hayan establecido por sí mismos como investigadores de vanguardia en el área de la regeneración de tejidos, haciéndolo con cuerpo científico continuo, sustentado en la investigación con aplicaciones clínicas y centrado en el ámbito de la regeneración de los tejidos en relación con el sistema musculoesquelético ${ }^{48}$.

Urist se mantuvo activo en la UCLA hasta su muerte, que ocurrió el 4 de febrero de 2001 a la edad de 86 años. Según una nota publicada el 7 de febrero de 2001 en las noticias UCLA Newsroom dentro de su apartado Health+Behavior, el entonces decano de la Escuela de Medicina y rector de Ciencias Médicas, el Dr. Gerald S. Levey, mencionó que Urist había dedicado su vida a la ortopedia, también que muchos avances en el campo habían sido resultado de su trabajo e investigación clínica, y que aquel día de su muerte habían perdido a un gran médico y científico ${ }^{49}$. Ese mismo año, el Dr. Rüdiger von Versen, entonces editor en jefe de la revista Cell and Tissue Banking, mencionó que el sentido de Urist sobre lo que era correcto y adecuado, así como su capacidad de pensar en el contexto más amplio, siempre salvaguardaron su juicio fiable, además de que poseía una energía y capacidad para entusiasmar de manera inquebrantable hasta el final ${ }^{15}$. En el año 2009, Richard Brand, siendo editor de la revista Clinical Orthopedics and Related Research, decidió publicar un simposio titulado "Symposium: Tribute to Dr. Marshall Urist: Musculoskeletal Growth Factors» ${ }^{50}$, en donde se incluyeron, además de su fotografía en la portada del volumen, una breve biografía y tres de los artículos considerados como más importantes (antes mencionados) ${ }^{30,32,34}$ (Fig. 4).

Tal vez sean pocos ya los elogios o reconocimientos que pueden agregarse al héroe de guerra, escritor, investigador, médico ortopedista, profesor,

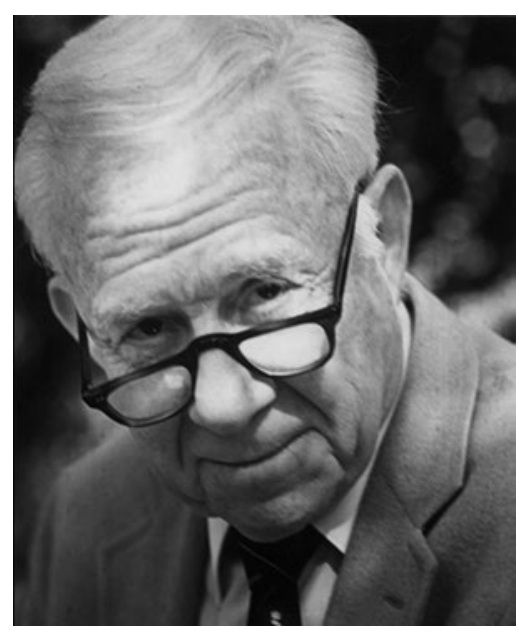

Figura 4. Prof. Dr. Marshall R. Urist (1914-2001). Fotografía incluida en la portada del volumen 467, No.12 de la revista Clinical Orthopaedics and Related Research, del año 2009, dedicado a su vida, investigaciones y aportaciones. Reproducida con autorización de Springer Customer Service Centre GmbH: Springer, Clinical Orthopaedics and Related Research, Marshall R. Urist, 1914-2001, Brand RA, COPYRIGHT 2017.

académico, pionero y ser humano; sin embargo, creo que es necesario conocerle a través de esta biografía en la comunidad iberoamericana en nuestra lengua, para así sumarnos, por los temas que nos competen, al reconocimiento de su labor y herencia científica a más de 100 años de su nacimiento. Como dijo el dramaturgo alemán Bertolt Brecht en una canción titulada In Praise of the Fighters, compuesta en 1931 para la obra teatral The Mother, «hay hombres que luchan un día y son buenos. Hay otros que luchan un año y son mejores. Hay quienes luchan muchos años y son muy buenos. Pero los hay quienes luchan toda la vida: esos son los imprescindibles».

\section{Bibliografía}

1. Bick EM. American orthopedic surgery: the first 200 years. Bull N Y Acad Med. 1976;52:293-325.

2. Eckardt JF. Historical notes. Marshall R. Urist, M.D. 1914-2001. UCLA/ Orthopaedic Hospital. Department of Orthopaedic Surgery. Alumni Bulletin 2014. p. 5-6.

3. Urist MR. The Journal of NIH Research 1997;9(1-6).

4. Urist MR. Bone morphogenetic protein: the molecularization of skeletal system development. J Bone Min Res. 1997;12:343-6.

5. Finerman GA. Marshall R. Urist, MD: 1914-2001. J Bone Joint Surg Am. 2001;83:1611.

6. Urist MR, Ries L, Quigley TB. Improved methods of management of compound fractures of leg. US Army Med Dept Cull. 1947;7:396-8.

7. Urist MR. Injuries to the hip joint. Traumatic dislocations incurred chiefly in jeep accidents in World War II. Am J Surg. 1947;74:586-97.

8. Rogers WA. Book reviews. Surgery in World War II. Orthopaedic surgery in the European theater of operations. Mather Cleveland, M.D. Washington, Office of The Surgeon General, Department of the Army, 1956. J Bone Joint Surg Am. 1957;39:477-8.

9. Urist MR. The management of battle-incurred compound fractures in the region of the hip joint (The Wellcome Award Essay, 1947). Mil Surg. 1947; 101:467-89.

10. Urist MR, Ries L, Quinley TB. A plaster traction splint for compound comminuted fractures of the tibia and fibula. Surgery. 1948;23:801-5. 
11. Urist MR. Fractures of the acetabulum. The nature of the traumatic lesions, treatment, and two year end results. Ann Surg. 1948;127:1150-64.

12. Urist MR. Fracture dislocation of the hip joint. The nature of the traumatic lesion, treatment, late complications and results. J Bone Joint Surg. 1948;30:699-727.

13. Urist MR, Quingley TB. Use of skeletal traction for mass treatment of compound fractures. A summary of experience with 4290 cases during Worl War II. AMA Arch Surg. 1951;63:834-44.

14. Reiddi H. Marshall R. Urist: a renaissance scientist and orthopaedic surgeon. J Bone Joint Surg. 2003;85-A(Suppl 3):3-7.

15. Von Versen R. In Memoriam Marshall R. Urist (1914-2001). Cell Tissue Bank. 2001;2:185-6

16. Brand RA. 50 years ago in CORR. Physiologic basis of bone-graft surgery. Marshall R. Urist MD. CORR 1953;1:207-216. Clin Orthop Relat Res. 2008;466:2015-6.

17. Finerman G. Marshall R. Urist MD, orthopaedic surgery: Los Angeles 1914-2001 professor. Universisty of California In Memoriam; 2001.

18. Urist MR, Budy AM, McLean FC. Factors influencing the reaction of the mammalian skeleton to estrogens. Trans Con for Metabolic Aspects of Convalescence. 1948:17:79-105.

19. Urist MR, Budy AM, McLean FC. Species differences in the reaction of the mammalian skeleton to estrogens. Proc Soc Exp Biol Med. 1948;68:324-6.

20. Urist MR, Budy M, McLean FC. Endosteal-bone formation in estrogen-treated mice. J Bone Joint Surg Am. 1950;32:143-62.

21. Delta-Kappa Award. AAOS 1950. Disponible en: http://www.aaos.org/ research/committee/research/Kappa/kappa_winners.asp\#1950

22. Crelin ES. Book review. Bone: an introduction to the physiology of skeletal tissue. Yale J Biol Med. 1961;34:150.

23. Brash JC. Book review. Bone. An introduction to the physiology of skeletal tissue. Journal of Anatomy. 1956;90(Pt 1):155-6.

24. Greenwald SA, Heim $\mathrm{C}$, Reddi $\mathrm{H}$, et al. The American Academy of Orthopaedic Surgeons. Section C. Academy News. The 2004 Annual Meeting Edition of the AAOS Bulletin. Today's News. Thursday, March 11, 2004. Profile of Orthopaedic Accomplishment: Marshall R. Urist, MD. Disponible en: http://www2.aaos.org/acadnews/2004news/c11-4.htm

25. Urist MR. Bone: formation by autoinduction. Science. 1965;150:893-9.

26. ORS. Marshall R. Urist, MD Award. Disponible en: http://www.ors.org/ blog/2000/11/21/urist-award.

27. Urist MR. Physiologic basis of bonegraft surgery. Clin Orthop Relat Res. 1953;1:207-16.

28. Brand RA. 50 years ago in CORR: physiologic basis of bone-graft surgery. Marshall R. Urist MD CORR 1953;1:207-216. Clin Orthop Relat Res. 2008;466:2015-6.

29. Urist MR. A morphogenetic matrix for differentiation of bone tissue. Calcif Tissue Res. 1970;(Suppl):98-101.

30. Symposium: Tribute to Dr. Marshall Urist: Muskuloskeletal growth factors. The classic: A morphogenetic matrix for differentiation of bone tissue. Marshall R. Urist MD. Clin Orthop Relat Res. 2009;467:3068-70.

31. Nogami H, Urist MR. A morphogenetic matrix for differentiation of cartilage in tissue culture. Proc Soc Exp Biol Med. 1970;134;530-5.
32. Symposium: Tribute to Dr. Marshall Urist: Muskuloskeletal growth factors. The classic: A morphogenetic matrix for differentiation of cartilage in tissue culture. Hiroshi Nogami and Marshall R. Urist, MD. Clin Orthop Relat Res. 2009;467:3051-5.

33. Urist MR, Strates BS. Bone morphogenetic protein. J Dent Res. 1971;50:1392-406

34. Symposium: Tribute to Dr. Marshall Urist. Musculoskeletal growth factors. The classic: Bone morphogenetic protein. Marshall R. Urist MD, Basil S. Strates. Clin Orthop Relat Res. 2009;467:3051-62.

35. Brand RA. Symposium: Tribute to Dr. Marshall Urist: Musculoskeltal Growth Factors. Marshall R. Urist, 1914-2001. Clin Orhtop Relat Res. 2009;467:3049-50.

36. Urist MR, DeLange RJ, Finerman GAM. Bone cell differentiation and growth factors: induced activity of chondro-osteogenic DNA. Science. 1983;220:680-6.

37. Urist MR. Bone: formation by autoinduction. Science. 1965;150:893-9.

38. Urist MR, Silverman BF, Buring K, etal. The bone induction principle. Clin Orthop. 1967;53:243-83.

39. Dubuc FL, Urist MR. The accessibility of the bone induction principle in surface-decalcified bone implants. Clin Orthop. 1967;55:217-23.

40. Urist MR, Dowell TA, Hay PH, et al. Inductive substrates for bone formation. Clin Orthop. 1968;59:59-96.

41. Huggins $C B$, Urist MR. Dentin matrix transformation: rapid induction of alkaline phosphatase and cartilage. Science. 1970;167:896-8.

42. Sampath TK, Reddi AH. Dissociative extraction and reconstitution of extracelular matrix components involved in local bone differentiation. Proc Natl Acad Sci USA. 1981;78:7599-603.

43. Urist MR, Sato K, Brownell AG, et al. Human bone morphogenetic protein (hBMP). Proc Soc Exp Biol Med. 1983:173:194-9.

44. Urist MR, Huo YK, Brownell AG, et al. Purification of bovine bone morphogenetic protein by hydroxyapatite chromatography. Proc Natl Acad Sci USA. 1984;81:371-5.

45. Wozney JM, Rosen V, Celeste AJ, et al. Novel regulators of bone formation: molecular clones and activities. Science. 1988;242:1528-34.

46. Luyten FP, Cunningham NS, Ma S, et al. Purification and partial amino acid sequence of osteogenin, a protein initiating bone differentiation. J Biol Chem. 1989;264:13377-80.

47. Reddi AH. Bone morphogenetic proteins: an unconventional approach to isolation of first mammalian morphogens. Cytokine Growth Factor Rev. 1997;8:11-20.

48. Marshall R. Urist Award. Disponible en: http://www.ors.org/ blog/2000/11/21/urist-award/

49. Everly A. UCLA Newsroom Health+Behavior. Prominent orthopaedic surgeon Dr. Marshall Urist dies; taught and practiced medicine at UCLA since 1954. February 07, 2001. Disponible en: http://newsroom.ucla.edu/ releases/Prominent-Orthopedic-Surgeon-Dr-2124

50. Symposium: Tribute to Dr. Marshall Urist: Musculoskeletal Growth Factors. Clinical Orthopedics and Related Research. December 2009, Volume 467, Number 12, Copublished by Springer International Publishing AG with The Association of Bone and Joint Surgeons. ISSN: 0009-921X (Print) 1528-1132 (On Line). 